\title{
Análisis panbiogeográfico del género Galianthe subgénero Ebelia (Rubiaceae)
}

Panbiogeographic analysis of Galianthe subgenus Ebelia (Rubiaceae)

\author{
Javier E. Florentin ${ }^{1}$, Marcelo D. Arana ${ }^{2,3}$ \& Roberto M. Salas ${ }^{1}$
}

\begin{abstract}
Resumen
Galianthe (Rubiaceae: Spermacoceae) es un género con los subgéneros Galianthe y Ebelia, éste último comprende 11 especies neotropicales, con centro de diversidad se encuentra en Brasil. El análisis panbiogeográfíco enfatiza la importancia de la dimensión espacial de la biodiversidad para generar una comprensión más adecuada de los patrones y procesos evolutivos y enfoca los eventos vicariantes como la mayor fuerza de la fragmentación de biotas. Son escasos los estudios biogeográficos de Galianthe, por lo que el objetivo del presente trabajo es establecer los patrones de distribución de Ebelia mediante un análisis de trazos. Las especies poseen distribución mesoamericana, andina y chaqueña, cuatro de estas últimas son exclusivas de Brasil. Siete especies conforman un único trazo generalizado denominado "Paraná", que conecta las provincias biogeográficas Cerrado y Chaco del dominio Chaqueño y Atlántica, Bosque Paraná y Bosque de Araucaria, pertenecientes al dominio Paraná, ambos dominios se hallan íntegramente dentro de la subregión Chaqueña, región Neotropical. Debido a que el origen de la tribu Spermacoceae se ha establecido durante el Eoceno, la aridificación de Sudamérica generada por los eventos vicariantes como la orogenia andina e introgresiones marinas, posiblemente han favorecido la radiación adaptativa del subgénero Ebelia dentro de la subregión Chaqueña.
\end{abstract}

Palabras clave: panbiogeografía, región Neotropical, Spermacoceae, subregión Chaqueña.

\begin{abstract}
Galianthe (Rubiaceae: Spermacoceae) is a genus composed by the subgenus Galianthe and Ebelia, the latter includes 11 Neotropical species with a center of diversity located in Brazil. The panbiogeographic analysis emphasizes the importance of the spatial dimension of biodiversity to generate a better understanding of both, evolutionary patterns and processes. Panbiogeography focuses on vicariant events as the greatest force for the fragmentation of biota. There are few studies leading with the biogeography of Galianthe, so the aim of this study is to establish the distribution patterns of subgenus Ebelia by track analysis. The species belonging to Ebelia have Mesoamerican, Andean and Chacoan distribution, with four of the species endemic of Brazil. Seven species form a unique generalized track called "Parana", which connects the biogeographic provinces Cerrado and Chaco of Chacoan dominion, and Atlantic, Parana and Araucaria Forest, belonging to the Parana dominion. Both dominions belong to the Chacoan subregion of the Neotropical region. Because the origin of the Spermacoceae tribe has been established during the Eocene, the aridification of South America, generated by the vicariant events as Andean orogeny and marine introgression have possibly favoured the adaptive radiation of the subgenus Ebelia within the Chacoan subregion. Key words: panbiogeography, Neotropical region, Spermacoceae, Chacoan subregion.
\end{abstract}

\section{Introducción}

El género Galianthe Griseb. pertenece a la familia Rubiaceae, tribu Spermacoceae, la cual tiene una distribución pantropical y cuenta con alrededor de 61 géneros y 1235 especies (Groeninckx et al. 2009). Galianthe es considerado un taxón monofilético con límites morfológicos bien definidos y de reciente revisión taxonómica (Cabral \& Bacigalupo 1997; Cabral 2009); se caracteriza por tener inflorescencias laxas, flores heterostiladas, estigma bífido, granos de polen con doble retículo, frutos 1 -seminados y número básico $X=8$ (Cabral 2009); estos estados de caracteres

\footnotetext{
Instituto Botánica del Nordeste, IBONE, CONICET, Sargento Cabral 2131, cc 209, C.P. 3400, Corrientes, Argentina.

${ }^{2}$ Universidad Nacional de Río Cuarto, Depto. Ciencias Naturales, Facultad Cs. Exactas Fco-Qcas y Naturales, Orientación Plantas Vasculares, Ruta 36, Km 601, X5804ZAB, Río Cuarto, Córdoba, Argentina.

${ }^{3}$ Autor para correspondencia: marana@exa.unrc.edu.ar
} 
indican una posición basal en Spermacoceae s.S. (Robbrecht 1988; Salas et al. 2015). Es un género exclusivo del Neotrópico y está constituido por 50 especies divididas en dos subgéneros: 1) Galianthe subgen. Galianthe, exclusivamente sudamericano con 39 especies, y 2) Galianthe subg. Ebelia (Rchb.) E.L. Cabral \& Bacigalupo, cuya sinapomorfía es la presencia de mericarpos indehiscentes. Este subgénero está conformado por 11 especies, de las que se conoce someramente dos áreas de distribución, una sudamericana desde Bahía hasta Río Grande do Sul en Brasil, Paraguay, Uruguay y la rivera bonaerense en Argentina. La otra área de distribución está comprendida por México (Chiapas), Belice, Guatemala, Colombia, Ecuador y Perú (Cabral 2002). Las especies habitan principalmente en formaciones abiertas de pastizales, localmente nombradas como campos rupestres, campos bajos, distintos tipos de cerrado, campos de altitud, entre otras (Cabral \& Salas 2012).

La panbiogeografía es uno de los programas de investigación más importantes de la biogeografía actual, que enfatiza la importancia de la dimensión espacial (geográfica) de la biodiversidad, para permitir una comprensión más adecuada de los patrones y procesos evolutivos a partir de las distribuciones geográficas. El análisis básicamente consiste en marcar en mapas las localidades de distribución de los grupos monofiléticos y luego unir las localidades de registro de las especies mediante líneas de distancia menor, las cuales se denominan trazos individuales. Una vez obtenidos los trazos individuales, estos al superponerse indican la existencia de biotas ancestrales, que han sido fragmentadas por eventos vicariantes geológicos, tectónicos o climáticos. Cuando dos o más trazos generalizados confluyen en una misma área, ésta se considera un nodo, que se interpreta como una zona de convergencia tectónica y biótica (Christian et al. 2011; Grehan 2001; Hull 1988; Loaiza \& Morrone 2011; Morrone 2015). Los nodos pueden presentar alto endemismo y diversidad taxonómica, limites distribucionales, ausencia de ciertos taxones, incongruencia y convergencia de caracteres, e híbridos inusuales, entre otros atributos, por lo que su conocimiento es una de las contribuciones más importantes de la panbiogeografía (Morrone \& Escalante 2009).

Son escasos los estudios enfocados en la biogeografía de la tribu Spermacoceae, en especial de las especies de Galianthe; el único trabajo disponible es el de Iganci et al. (2011), en el que se analizaron los endemismos de los pastizales subtropicales de altura del sur de Brasil y encontraron en ellos a 3 especies de Galianthe: G. elegans E.L. Cabral, G. latistipula E.L. Cabral y G. reitzii E.L. Cabral, todas pertenecientes al subgénero Galianthe. Esto resalta una notable escasez en los conocimientos biogeográficos en Galianthe, en especial del subgénero Ebelia, por lo que el objetivo del presente trabajo es establecer los patrones de distribución de las especies de Galianthe subgénero Ebelia, mediante un análisis de trazos, en un marco panbiogeográfico, para establecer la homología biogeográfica primaria, que representa una hipótesis acerca de una historia biótica común de los taxones involucrados, basada en la congruencia distribucional.

\section{Materiales y Métodos}

Para establecer la distribución de las especies pertenecientes al subgénero Ebelia, se reunió la información proveniente de antecedentes bibliográficos, particularmente la revisión del genero Galianthe (Cabral 2002) y además se analizaron varios especímenes (más de 1200 entradas) conservados en los siguientes herbarios (acrónimos de acuerdo al Index Herbariorum): CORD, CTES, RCV, SI, HUFU, RB, UEC, HUCS, NY, HUEM, MO, HEPH, UB, ICN, MBM, FUEL, BHCB, SP, F, ESA, CEN, IAC, SPSF, HUEF. Para cada ejemplar se tomaron los datos provenientes de las etiquetas y se georreferenciaron cada uno de los especímenes, utilizando la herramienta Google Earth (2015).

Los datos obtenidos fueron volcados en planillas especialmente diseñadas para este fin, se tomaron los datos de localidad (en caso de tener con coordenadas), fenología (flor, fruto, fecha), ambiente (margen de río, interior de bosque, sotobosque, etc.), hábito, coleccionista, numero de colección y determinador. Dicha información se trasladó a una planilla de datos (Microsoft Office Excel). Para graficar los trazos individuales se utilizó el software DIVA-Gis Versión 4, Sistema de Información Geográfica para el Análisis de Datos de Distribución de Especies (Hijmans 2013), a partir de los datos georreferenciados se graficaron los trazos de acuerdo al criterio de distancia mínima. A partir de la superposición de los trazos individuales se establecieron los trazos generalizados y los nodos, los que fueron georreferenciados utilizando el mismo programa. Luego se superpusieron los trazos generalizados en un mapa representando el esquema delas provincias biogeográficas de la región Neotropical propuesto por Morrone (2006, 2014b). Los mapas resultantes fueron editados mediante Corel Photo Paint ${ }^{\circledR}$. 


\section{Resultados}

En los patrones de distribución de Galianthe subgénero Ebelia, conformado por 11 especies de distribución exclusivamente americana, se observa que sólo una, Galianthe angulata (Benth.) Borhidi (Fig. 1a), posee una distribución esencialmente mesoamericana, fuera de Sudamérica, en el sur de México, Guatemala y Belice (Cabral 2002). Las demás especies poseen una distribución esencialmente sudamericana, con tres especies restringidas al sector andino: G. bogotensis (Kunth) E.L. Cabral (Fig. 1b) y G. dichotoma (Willd. ex Roem. \& Schult.) E.L. Cabral \& Bacigalupo (Fig. 1c) en las provincias biogeográficas Cauca
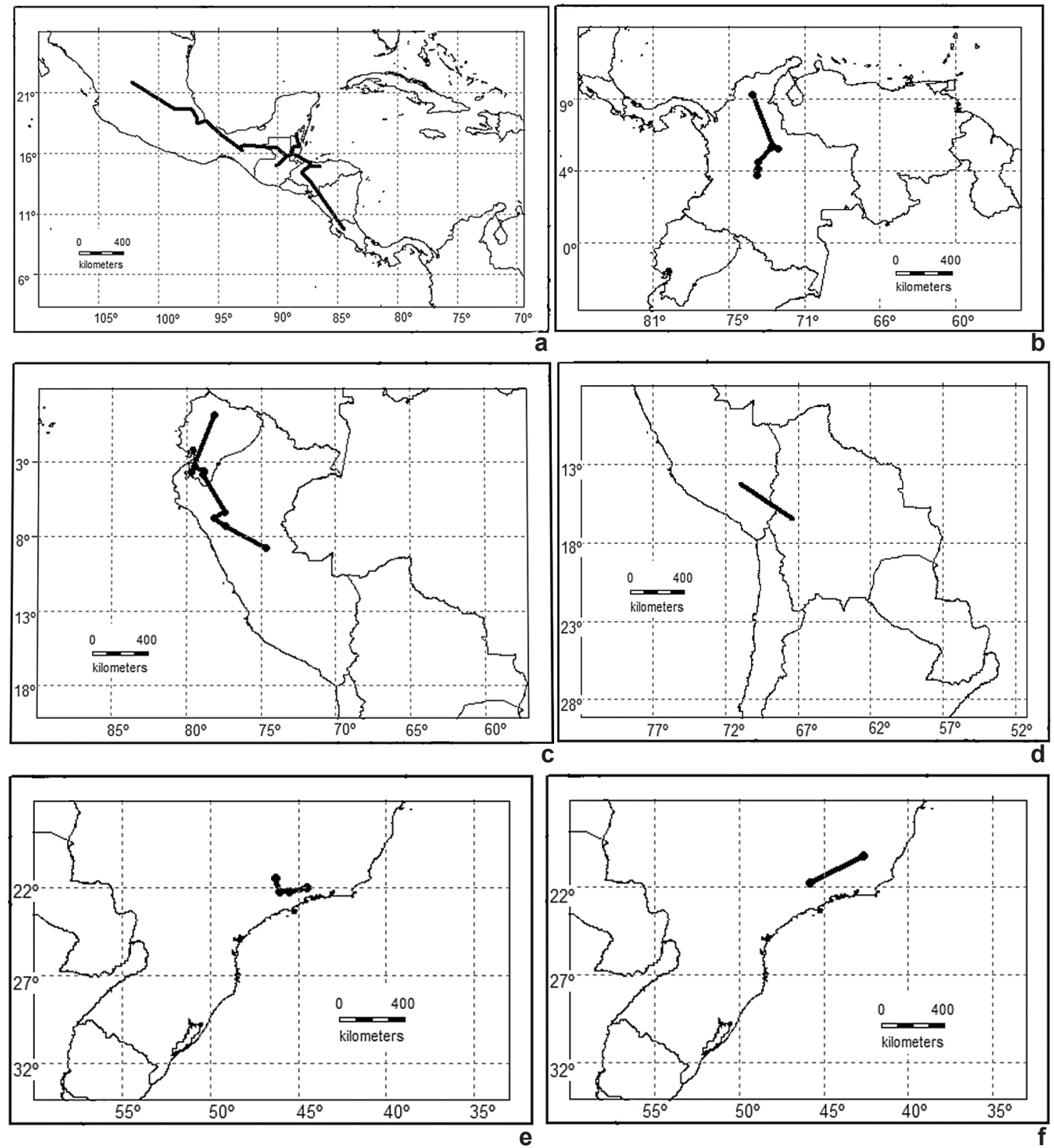

Figura 1 - Trazos individuales de las especies de Galianthe subgénero Ebelia - a. G. angulata; b. G. bogotensis; c. G. dichotoma; d. G. boliviana; e. G. vaginata; f. G. humilis.

Figure 1 - Individual tracks of the species of Galianthe subgenus Ebelia - a. G. angulata; b. G. bogotensis; c. G. dichotoma; d. G. boliviana; e. G. vaginata; f. G. humilis. 
y Magdalena, del Dominio Pacífico y en la provincia del Páramo, de la Zona de Transición Sudamericana, y G. boliviana E.L. Cabral, endémica de la provincia biogeográfica Puna, a partir de los 1800 s.m. (Fig. 1d). Las siete especies restantes son exclusivas de la Subregión Chaqueña: G. vaginata E.L. Cabral \& Bacigalupo (Fig. 1e), G. humilis E.L. Cabral \& Bacigalupo (Fig. 1f), G.
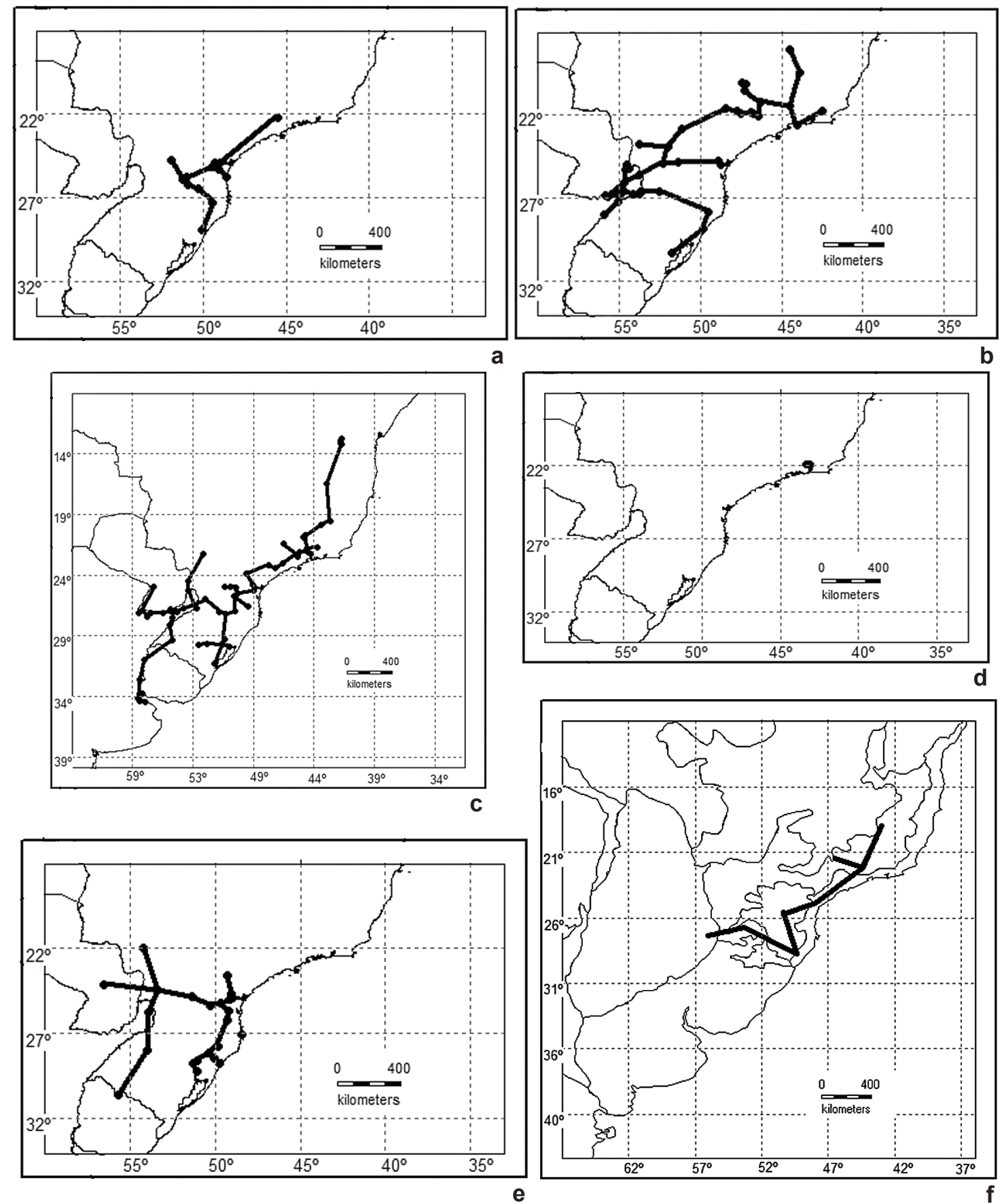

Figura 2 - Trazos individuales de las especies de Galianthe subgénero Ebelia - a. G. cymosa; b. G. hispidula; c. G. brasiliensis; d. G. polygonoides; e. G. dichasia; f. trazo generalizado "Paraná".

Figure 2 - Individual tracks of the species of Galianthe subgenus Ebelia - a. G. cymosa; b. G. hispidula; c. G. brasiliensis; d. G. polygonoides; e. G. dichasia; f. "Paraná" generalized track. 
cymosa (Cham.) E.L. Cabral \& Bacigalupo (Fig. 2a), G. hispidula (A. Rich. ex D.C.) E.L. Cabral \& Bacigalupo (Fig. 2b), G. brasiliensis (Spreng.) E.L. Cabral \& Bacigalupo (Fig. 2c), G. polygonoides E.L. Cabral \& Bacigalupo (Fig. 2d) y G. dichasia (Sucre \& C.G. Costa) E.L. Cabral (Fig. 2e), de las cuales $G$. polygonoides es una entidad endémica de la región de Rio de Janeiro, entre los 750-800 m s.m., caracterizada por el hábito trepador y una vaina estipular tubulosa, similar a una ocrea.

De acuerdo con la superposición gráfica de los trazos individuales, se puede observar un patrón distribucional principal formando un único trazo generalizado denominado "Paraná" (Fig. 2f), que conecta las siguientes provincias biogeográficas: Cerrado y Chaco del dominio Chaqueño y Atlántica, Bosque Paraná y Bosque de Araucaria, pertenecientes al dominio Paraná, ambos dominios se hallan íntegramente dentro de la subregión Chaqueña, región Neotropical. Es de destacar la ausencia de nodos, lo que indica que en la provincia biogeográfica Bosque Paraná no hay convergencia de biotas ancestrales del subgénero Ebelia.

\section{Discusión}

El patrón distribucional de Galianthe subgénero Ebelia establecido, que denominamos "Paraná", ya que queda incluido en su mayor parte en la provincia biogeográfica Bosque Paraná con pequeño sectores en el sur y sureste dentro de las provincias Cerrado y Chaco (dominio Chaqueño) y Atlántica y Bosque de Araucaria (dominio Paraná) dentro de la subregión Chaqueña. Este patrón distribucional principal posee un componente biótico formado por las especies: G. vaginata, $G$. humilis, G. cymosa y G. dichasia, características de las sabanas con árboles aislados que pertenecen a las provincias biogeográficas mencionadas, mientras que $G$. hispidula y $G$. brasiliensis habitan el interior de bosques y selvas debido a su forma de vida umbrófila. Este patrón distribucional es el reflejo de una única biota ancestral en esta área.

En el enfoque de la Panbiogeografía, los eventos tectónicos (vicarianza tectónica) y climáticos (vicariaza dinámica) constituyen la mayor fuerza de los cambios geográficos y fragmentación de las biotas ancestrales (Echeverry \& Morrone 2013). Las relaciones distribucionales de la biota del Cono Sur se vieron afectadas principalmente por fenómenos climáticos $\mathrm{y}$ geomorfológicos, que comenzaron durante la segunda parte del Neógeno (Crisci et al. 2001 \& Ponce et al. 2002). Durante el Cenozoico Tardío, el levantamiento de los Andes y el desarrollo de corrientes frías marítimas (en especial la de Humboldt) se han asociado con la diversificación de hábitats y oportunidades evolutivas para la biota (Axelrod et al. 1991). A partir del Mioceno y hasta el Plioceno, el clima se fue haciendo más árido debido al lento levantamiento de los Andes y a la corriente fría de Humboldt. La última fase de la orogenia andina en el Plioceno superior causó el solevantamiento de diversos sistemas serranos como las Sierras Pampeanas y Subandinas (Taylor 1991).

Se ha demostrado que previamente había una conexión entre las áreas selváticas sudamericanas, que conformaban una superficie boscosa continua (Nores 1992; Prado 1993; Prado \& Gibbs 1993; Pennington et al. 2000; Ganem et al. 2007; Arana et al. 2012; Yañez et al. 2014; Garcia et al. 2014) de la cual los bosques en galería a lo largo de los cursos de agua, por ejemplo de los ríos Bermejo, Pilcomayo, Paraguay y Paraná, constituirían relictos de una distribución más amplia de las selvas durante los períodos interglaciares del pasado reciente (Pleistoceno y Holoceno). En los períodos interglaciares, esta gran superficie boscosa fue gradualmente fragmentada en dos partes, una en el oeste (Yungas) y otra en el este (Paranaense) por el avance de la diagonal árida conformada por una gran planicie de bosques xerófilos chaqueños, provocando la consecuente vicarianza dinámica de los taxones que componen estos bosques húmedos subtropicales, que ocupan dos áreas disyuntas entre los $23^{\circ} \mathrm{S}$ y $29^{\circ} \mathrm{S}$, fragmentadas entre sí por el dominio Chaqueño (Morrone \& Coscarón 1996; Pennington et al. 2000; Katinas \& Crisci 2008), que habría sido más húmedo que actualmente (Van der Hammen 1974; Bigarella \& de Andrade-Lima 1982; Pennington et al. 2000). Esta secuencia de eventos está sustentada además por evidencias paleontológicas, paleoclimatológicas y geológicas que indican que un clima templado prevaleció en Sudamérica durante el Paleógeno y fue seguido de un enfriamiento y aridificación en el Oligoceno y Mioceno, proceso completado por el alzamiento de los Andes (Romo \& Morrone 2011; Simões et al. 2012). Estos patrones de distribución están estrechamente ligados con las glaciaciones del Pleistoceno, que afectaron unos dos tercios de los bosques de América del Sur austral, produciendo desplazamientos, compresión y extinciones de las biotas (Villagrán \& Hinojosa 1997). El dominio Chaqueño es una gran cuenca sedimentaria limitada por los Andes y el cratón brasileño, comprende un área de aproximadamente $840.000 \mathrm{~km}^{2}$, la que 
puede ser definida como una serie de grandes abanicos aluviales generados por los ríos que la atraviesan (Iriondo 1993; Argollo Bautista \& Iriondo 2008). Geomorfológicamente, esta Región es el resultado de una masiva acumulación de sedimentos cuaternarios sobre el hundido escudo Precámbrico Brasileño (Prado 1993). Asimismo, a lo largo del Cuaternario Superior, ocurrieron importantes cambios climáticos, pasando desde climas fríos y secos como en el Último Máximo Glaciar (UMG), hasta climas más subtropicales y tropicales (Iriondo \& García 1993). En consecuencia, esta región tuvo un tiempo de formación breve, con un crecimiento derivado de continuos aportes superficiales de materiales transportados en su mayoría por el viento, con modificaciones fluviales (Molina 2006).

Desde un punto de vista biogeográfico evolutivo, el Dominio Chaqueño puede ser considerado un ambiente secundario. Considerando que los patrones climáticos actuales de Sudamérica comenzaron a establecerse a partir del Mioceno, se ha postulado una edad pliocena para la identidad biogeográfica del Chaco (Iriondo 1992). A lo largo de la llanura chaqueña, en correlación con la aridez climática y edáfica, la vegetación cambia desde bosques xerofíticos y matorrales en el noreste (Caatingas, Cerrado), a bosques semideciduos entremezclados con extensas sabanas y palmares en el sureste (Pampa). La vegetación de la orilla de los principales ríos y sus afluentes occidentales es, generalmente, un bosque en galería (Morello 1967; Morello \& Adamoli 1968, 1974; Prado 1993). Se ha sugerido que el patrón de la vegetación Chaqueña es el resultado de la dinámica aluvial durante el Pleistoceno-Holoceno de la Región, la migración intensiva de los cinturones fluviales de los ríos principales (Iriondo 1995; Morelo \& Matteucci 1999) y la gran introgresión marina del Mioceno formada por el Mar Paranaense, controlada por eventos tectónicos (Hernandez et al. 2005; Lovejoy et al. 2006).

Debido a que el origen de la tribu Spermacoceae se ha establecido hace unos 44,3 millones de años atrás (Bremer \& Eriksson 2009), durante el Eoceno del período Paleógeno (Era Cenozoica), los eventos vicariantes mencionados previamente muy posiblemente han favorecido la radiación adaptativa dentro de las unidades biogeográficas de la Subregión Chaqueña (Werneck 2011), como podría ser el caso de las especies de Galianthe, en los dominios Chaqueño y Parana; ya que son plantas que poseen adaptaciones a ambientes xéricos y suelos arenosos, lateríticos o con afloramientos rocosos y pastizales abiertos, como ser la presencia de xilopodio, cutícula gruesa y pequeña superficie foliar, lo que les permitió prosperar en los ambientes cuaternarios abiertos, xéricos y cálidos de la Subregión Chaqueña. Esta conexión del trazo generalizado vinculando los dominios Chaqueño y Paraná apoya lo obtenido por otros autores mediante un análisis cladístico de áreas utilizando diversos taxones, como artrópodos, vertebrados y otras angiospermas y cuya vicarianza se produjo principalmente durante el Paleoceno (Nihei et al. 2004; Morrone 2014a). Por otra parte, las zonas montañosas y serranas contribuyen al establecimiento y supervivencia de las poblaciones de plantas en general por la gran heterogeneidad de hábitats y microclimas que generan (Morán 1995; Arcand \& Ranker 2008), teniendo en cuenta la particularidad de los microambientes de las serranías inmersas en la Subregión Chaqueña, las mismas se desempeñarían como islas biogeográficas dentro de la gran planicie formada por la cuenca sedimentaria chaqueña, lo que favorecería la vicarianza y especiación dentro del género Galianthe (y la tribu Spermacoceae en general), ya que se han encontrado taxones endémicos de esta región, como Galianthe polygonoides, que en general habita en pastizales y campos abiertos de altura, a partir de los $750 \mathrm{~m}$ s.m. Aunque algunos autores (Costa 2003; Nihei \& Carvalho 2007) expresan que no es posible postular una única hipótesis sobre la distribución de la biota Neotropical, el patrón biogeográfico de Ebelia obtenido, combinado con la edad de aparición del grupo al que pertenece, obtenida por análisis moleculares y calibración con fósiles (Bremer \& Eriksson 2009), está acorde con la secuencia de eventos vicariantes que se ha propuesto previamente como procesos que han conducido a la diversificación de la biota Neotropical, y apoya la regionalización biogeográfica propuesta por Morrone (2014a,b), sobre la base de análisis biogeográficos cladísticos utilizando otros taxones, en donde el dominio Paraná y Chaqueño están estrechamente relacionados y pertenecen a la subregión Chaqueña.

\section{Agradecimientos}

Al Consejo Interuniversitario Nacional de Argentina por la beca CIN otorgada, A la Dra. Elsa Cabral (IBONE) por sus valiosos consejos y a la Dra. Evangelina Natale (UNRC) por su ayuda. A la editora Dra. Daniela Zappi por sus valiosas sugerencias. También se desea agradecer al Instituto Botánica del Nordeste (IBONE) y a Universidad Nacional de Rio Cuarto (UNRC) por facilitar este estudio en sus instalaciones. 


\section{Referencias}

Arana, M.D.; Morrone, J.J.; Ganem, M.A.; Luna, M.L.; Ramos Giacosa, J.P. \& Giudice, G. 2012. Diversidad y análisis panbiogeográfico de las licofitas (Embryopsida: Lycopodiidae) del Parque Nacional Calilegua, Jujuy, Argentina. Iheringia (Série Botanica) 67: 177-188.

Arcand, N.N. \& Ranker, T.A. 2008. Conservation biology. In: Ranker, T.A. \& Haufler, C.H. (eds.). The biology and evolution of ferns and lycophytes. Cambridge University Press, New York. Pp. 257283.

Argollo Bautista, J. \& Iriondo, M. 2008. El Cuaternario de Bolivia y regiones vecinas. Museo Provincial de Ciencias Naturales "Florentino Ameghino", Santa Fe. 280p.

Axelrod, D.I.; Kalin Arroyo, M.T. \& Raven, P.H. 1991. Historical development of temperate vegetation in the Americas. Revista Chilena de Historia Natural 64: 413-446.

Bigarella, J.J. \& de Andrade-Lima, D. 1982. Paleoenvironmental changes in Brazil. In: Prance, G.T. (ed.). Biological diversification in the tropics. Columbia University Press, New York. Pp. 27-40.

Bremer, B. \& Eriksson, O. 2009. Time tree of Rubiaceae: phylogeny and dating the family, subfamily, and tribes. International Journal of Plant Science 170: 766-793.

Cabral, E.L. 2002. Revisión del género Galianthe Griseb. (Rubiaceae). Tesis Doctoral. Universidad Nacional del Nordeste, Corrientes. 400p.

Cabral, E.L. 2009. Revision Sinóptica de Galianthe Subgen. Galianthe (Rubiaceae: Spermacoceae), con una sección nueva. Annals of the Missouri Botanical Garden 96: 27-60.

Cabral, E.L. \& Bacigalupo, N.M. 1997. Revisión del género Galianthe subg. Ebelia stat. nov. (Rubiaceae-Spermacoceae). Annals of the Missouri Botanical Garden 83: 857-877.

Cabral, E.L. \& Salas, R.M. 2012. Galianthe. In: Forzza, R.C. et al. (org.). Instituto de Pesquisas Jardim Botânico do Rio de Janeiro. Catálogo de Plantas e Fungos do Brasil 2: 1590-1591.

Costa, L.P. 2003. The historical bridge between the Amazon and the Atlantic Forest of Brazil: a study of molecular phylogeography with small mammals. Journal of Biogeography 30: 71-86.

Crisci, J.V.; Freire, S.E.; Sancho, G. \& Katinas, L. 2001. Historical biogeography of the Asteraceae from Tandilia and Ventania Mountain ranges. Caldasia 23: 21-41.

Echeverry, A. \& Morrone, J.J. 2013. Generalized tracks, area cladograms and tectonics in the Caribbean. Journal of Biogeography 40: 1619-1637.

García, M.V.; Prinz, K.; Barrandeguy, M.E.; Miretti, M. \& Finkeldey, R. 2014. A unifying study of phenotypic and molecular genetic variability in natural populations of Anadenanthera colubrina var. cebil from Yungas and Paranaense biogeographic provinces in Argentina. Journal of Genetics 93: $1-10$.

Ganem, M.A.; Giudice, G.E.; Luna, M.L. \& de la Sota, E.R. 2007. Revisión del grupo "Asplenium squamosum" en América. Candollea 62: 149-156.

Google Earth. 2015. Disponible en $<$ http://www.google earth.com.ar/>. Acceso en marzo 2015.

Greham, J.R. 2011. Panbiogeografía y la geografía de la vida. In: Llorente, J. \& Morrone, J.J. (eds.). Introduccion a la biogeografia en Latinoamerica: teorias, conceptos, metodos y aplicaciones. Universidad Autónoma de México, México-D.F. Pp. 181-195.

Hernández, R.M.; Jordan, T.E.; Farjat, A.D.; Echevarría, L.; Idleman, B.D. \& Reynolds, J.H. 2005. Age, distribution, tectonics and eustatic controls of the Paranense and Caribbean marine transgressions in southern Bolivia and Argentina. Journal of South American Earth Sciences 19: 495-512.

Hijmans, R.J. 2013. DIVA-GIS, a geographic information system for the analysis of biodiversity data. Versión 7.5. Disponible en $<$ http://www.diva.gis.org $>$. Acceso en marzo 2015.

Hull, D.L. 1988. Science as a process: an evolutionary account of the social and conceptual development of science. University of Chicago Press, Chicago. $586 \mathrm{p}$.

Iganci, J.R.; Heiden, G.; Miotto, S.T.S. \& Pennington, R.T. 2011. Campos de Cima da Serra: the brazilian subtropical highland grasslands show an unexpected level of plant endemism. Botanical Journal of the Linnean Society 167: 378-393.

Iriondo, M.H. 1993. Geomorphology and late Quaternary of the Chaco (South America). Geomorphology 7: 289-303.

Iriondo, M. \& Garcia, N. 1993. Climatic variations in the Argentine plains during the last 18.000 years. Palaeogeography, Palaeoclimatology, Palaeoecology. An International Journal for the Geo-Sciences 141: 209-220.

Katinas, L. \& Crisci, J. 2008. Reconstructing the biogeographical history of two plant genera with different dispersion capabilities. Journal of Biogeography 35: 1374-1384.

Loaiza, C.R.S. \& Morrone, J.J. 2011. Análisis panbiogeográfico de algunas Cactaceae del Ecuador. Gayana Botánica 68: 220-225.

Lovejoy, N.R.; Albert, J.S. \& Crampton, W.G.R. 2006. Miocene marine incursions and marine/freshwater transitions: evidence from neotropical fishes. Journal of South American Earth Sciences 21: 5-13.

Molina, A.M. 2006. Flora Chaqueña Argentina (Formosa, Chaco y Santiago del Estero), Familia Gramíneas. Colección Científica del INTA 23: i-viii+1-848

Moran, R.C. 1995. The importance of mountains to Pteridophytes, with emphasis on Neotropical 
montane forests. In: Churchill, S.P. (ed.). Biodiversity and conservation of Neotropical montane forests. New York Botanical Garden, New York. Pp. 359-363.

Morello, J. 1967. Bases para el estudio fitoecológico de los grandes espacios (el Chaco argentino). Ciencia e Investigación 23: 252-267.

Morello, J. \& Adámoli, J. 1968. Las grandes unidades de vegetación y ambiente del Chaco argentino. Primera parte: objetivos y metodología. Serie Fitogeográfica 10. INTA, Buenos Aires. 125p.

Morello, J. \& Adámoli, J. 1974. Las grandes unidades de vegetación y ambiente del Chaco Argentino. Segunda parte: vegetación y ambiente de la Provincia del Chaco. Serie Fitogeográfica 13. INTA, Buenos Aires. 131p.

Morello, J. \& Matteucci, S.D. 1999. Biodiversidad y fragmentación de los bosques en la Argentina. In: Matteucci, S.D.; Solbrig, O.T.; Morello, J. \& Halffter, G. (eds.). Biodiversidad y uso de la tierra. Conceptos y ejemplos de Latinoamérica. Eudeba-Unesco, Buenos Aires. Pp. 463-498.

Morrone, J.J. 2006. Biogeographic areas and transition zones of Latin America and the Caribbean islands based on panbiogeographic and cladistic analyses of the entomofauna. Annual Review of Entomology 51: 467-494.

Morrone, J.J. 2014a. Cladistic biogeography of the Neotropical region: identifying the main events in the diversification of the terrestrial biota. Cladistics 30: 202-214.

Morrone, J.J. 2014b. Biogeographical regionalisation of the Neotropical region. Zootaxa 3782: 1-110.

Morrone, J.J. 2015. Track analysis beyond panbiogeography. Journal of Biogeography 42: 413-425.

Morrone, J.J. \& Coscarón, M.C. 1996. Distributional patterns of the American Peiratinae (Heteroptera: Reduviidae). Zoologische Mededelingen Leiden 70: 1-15.

Morrone, J.J. \& Escalante, T. 2009. Diccionario de biogeografía. Las prensas de Ciencias, México-D.F. 230p.

Nihei, S.S. \& Carvalho, C.J.B. 2004. Taxonomy, cladistics and biogeography of Coenosopsia Malloch (Diptera, Anthomyiidae) and its significance to the evolution of anthomyiids in the Neotropics. Systematic Entomology 29: 260-275.

Nihei, S.S. \& Carvalho, C.J.B. 2007. Systematics and biogeography of Polietina Schnabl and Dziedzicki (Diptera, Muscidae): neotropical area relationships and Amazonia as a composite area. Systematic Entomology 32: 477-501.
Nores, M. 1992. Bird speciation in subtropical South America in relation to forest expansion and retraction. The Auk 109: 346-357.

Pennington, R.T.; Prado, D.E. \& Pendry, C.A. 2000. Neotropical seasonally dry forests and quaternary vegetation changes. Journal of Biogeography 27 : 261-273.

Ponce, M.; Mehltreter, K. \& de la Sota, E.R. 2002. Análisis biogeográfico de la diversidad pteridofítica en Argentina y Chile continental. Revista Chilena de Historia Natural 75: 703-717.

Prado, D.E. 1993. What is the Gran Chaco vegetation in South America? I. A review. Contribution to the study of flora and vegetation of the Chaco V. Candollea 48: 145-172.

Prado, D.E. \& Gibbs, P.E. 1993. Patterns of species distributions in the dry seasonal forests of South America. Annals of the Missouri Botanical Garden 80: 902-927.

Robbrecht, E. 1988. Tropical woody Rubiaceae. Opera Botanica Belgica 1: 1-272.

Romo, A. \& Morrone, J.J. 2011. Track analysis of the Neotropical Entimini (Coleoptera: Curculionidae: Entiminae). Revista Brasileira de Entomologia 55: 313-316.

Salas R.M.; Viana, P.L.; Cabral, E.L.; Dessein, S. \& Jansen, S. 2015. Carajasia (Rubiaceae), a new and endangered genus from Carajás mountain range. Pará, Brazil. Phytotaxa 206: 14-29.

Simões, F.L.; Augusto Ferrari, A. \& Grazia, J. 2012. Is Elsiella Froeschner, 1981 a valid genus? (Hemiptera: Heteroptera: Pentatomidae: Pentatominae). Zootaxa 3238: 39-48.

Taylor, D.W. 1991. Paleobiogeographic relationships of Andean angiosperms of Cretaceous to Pliocene age. Palaeogeography, Palaeoclimatology, Palaeoecology 88: 69-84.

Van der Hammen, T. 1974. The Pleistocene changes of vegetation and climate in tropical South America. Journal of Biogeography 1: 3-26.

Villagrán, C. \& Hinojosa, L.F. 1997. Historia de los bosques de Sudamérica II: análisis fitogeográfico. Revista Chilena de Historia Natural 70: 241-267.

Werneck, F.P. 2011. The diversification of eastern South American open vegetation biomes: historical biogeography and perspectives. Quaternary Science Reviews 30: 1630-1648.

Yañez, A.; Arana, M.D.; Marquez, G.J. \& Oggero, A. 2014. The genus Dennstaedtia Bernh. (Dennstaedtiaceae) in Argentina. Phytotaxa 174: 69-81. 